\title{
Micromachined Gyroscopes: Challenges, Design Solutions, and Opportunities
}

\author{
Andrei M. Shkel \\ MicroSystems Laboratory \\ Department of Mechanical and Aerospace Engineering \\ University of California, Irvine, CA, USA
}

\begin{abstract}
Micromachined gyroscopes are probably the most challenging type of transducers ever attempted to be designed in micro-world. A nail-size dynamic system integrated with control electronics on the same silicon chip (Fig. 1(a)) is designed to be a very sensitive sensor which is potentially able to detect maneuvers and motions beyond human perception. Along with exciting opportunities which MEMS gyroscopes could bring to everyday life, the miniaturization introduces many new technical challenges. Multi-degree of freedom dynamics, sensitivity to fabrication imperfections, dynamic instability, limited control resources - all these raise a number of fundamentally challenging issues in the design, analysis, and control of micromachined gyroscopes. In this paper, we summarize principles of operation, review recent research and development efforts, and discuss potential applications and the future market of silicon based micromachined gyroscopes.
\end{abstract}

Keywords: MEMS Gyroscopes, Inertial Sensors, Gyroscope Applications

\section{INTRODUCTION}

Truly low-cost inertial sensors have been a goal of the industry for many years. Until recently, the high cost of precision inertial sensors has precluded their use in automotive applications, consumer electronics, robotics, and a wide range of military applications.

Recent advances in silicon micromachining technology have raised the possibility of revolutionizing the field of inertial instruments by providing inexpensive, miniature gyroscopes, to address market needs for low-cost medium performance inertial instruments.

Micromachined accelerometers have been extremely successful in high volume and low-cost automotive applications such as air bags, vehicle stabilization systems and active suspensions. Micro-gyroscopes are projected to exhibit a similar success. While MEMS accelerometers are relatively mature, current MEMS gyroscope prototypes are technically immature and unreliable. World-wide, many universities, research centers, and industrial laboratories make efforts to develop concepts and fabrication technologies that may improve the performance and reliability of micromachined gyroscopes.

The goal of this paper is to review current research and development efforts in the area of miniaturized inertial instruments and highlight challenges in development of reliable MEMS gyroscopes.

\section{APPLICATIONS}

There is a wide range of potential applications for inexpensive gyroscopes (Fig. 2(a)). Until recently, the high-cost, size, weight, and power consumption of the conventional gyroscopes (Fig. 1(b)) precluded their use in automotive applications, consumer electronic, computer peripherals, and Global Positioning Systems (GPS) augmentation. As soon as the first family of inexpensive inertial sensors is on the market, they will revolutionize the way we think about consumer electronics, personal navigation, and appliances. Without doubts, micromachined gyroscopes will render new markets in the automotive, aerospace, defense, industrial, commercial, and medical industries.

Further author information: (Send correspondence to A.M.S.: E-mail: ashkel@uci.edu) Web: http://mems.eng.uci.edu 

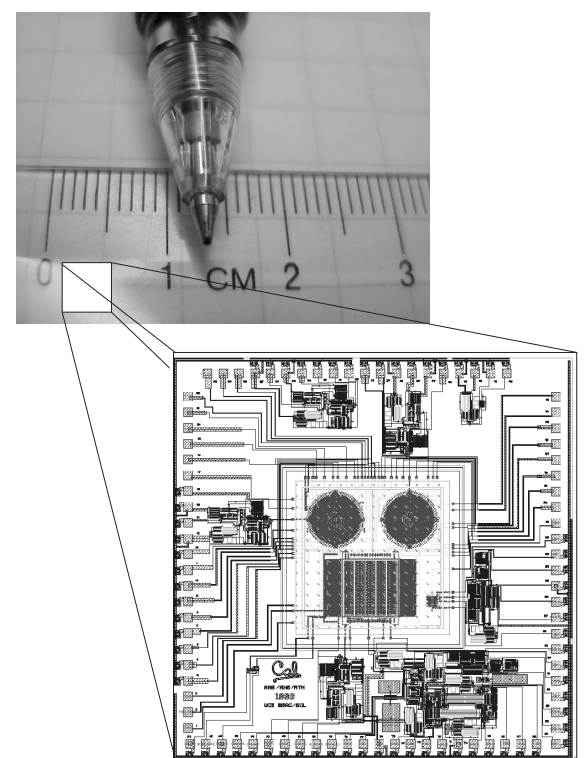

(a)

\section{Conventional}

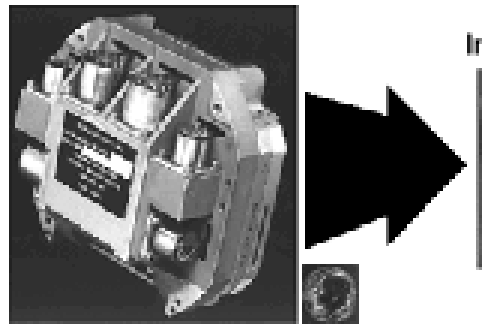

Mass: 1587.5 grams

Size: $15 \mathrm{~cm} \times 8 \mathrm{~cm} \times 5 \mathrm{~cm}$

Power: $35 \mathrm{~W}$

Survivability: $35 \mathrm{~g}$ 's

Cost: $\$ 20,000$
MEMS

Inertial Measurement Unit
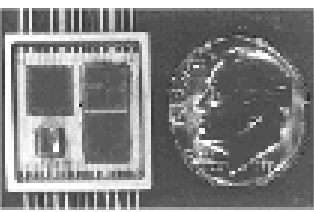

Mass: 10 grams

Size: $2 \mathrm{~cm} \times 2 \mathrm{~cm} \times 0.5 \mathrm{~cm}$

Power: $\sim 1 \mathrm{~mW}$

Cost: $\$ 500$

(b)

Figure 1. (a)Fingernail sized gyro module includes three gyroscopes integrated with on-chip control electronics. The chip is being fabricated in Sandia's iMEMS technology; (b) Conventional vs. MEMS inertial measurement unit. ${ }^{1}$ Micromachined gyroscopes will lead to orders of magnitude reduction in cost, size, weight, and power consumption compare to their conventional counterparts

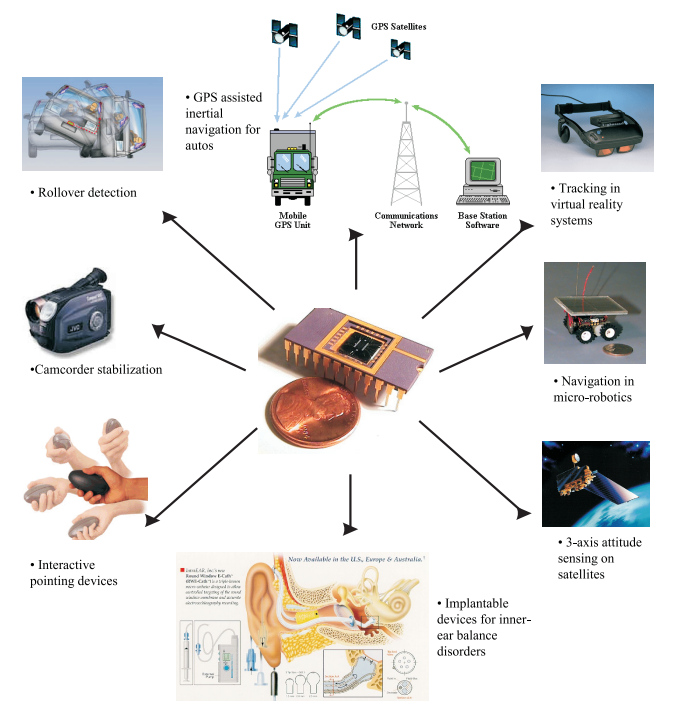

\section{Automotive Sensor Applications}

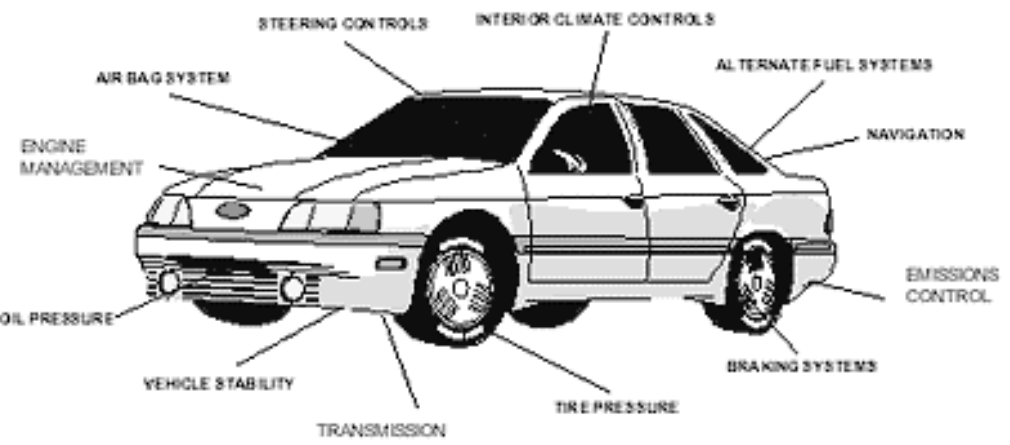

Current cars have 50.85 sensorsivelicle. Future projections are $100 *$ potential sensorsivehicle. Each sensor needs/will need read-out circuitry.

Figure 2. (a) There is an emerging need for low-cost gyroscopes in automotive industry. Other industries, including aerospace, defense, consumer electronics, and medicine will greatly benefit as well; (b) Application of sensors in automotive industry

\subsection{Automotive Application}

The automotive industry is a major commercial driver for development of MEMS-based inertial measurement products.

There is a large consumer demand for ride stabilization systems, rollover detection units, antilock braking systems (ABS) as well as other vehicle safety systems. Currently, cars are equipped with approximately 50-85 sensors per vehicle (Figure 2(b)). It the projected, that the number of sensors per vehicle will double within the next few years, and micromachined gyroscopes along with accelerometers are going to be the key vehicle safety sensors. 
As an example, an array of sensors including the yaw gyroscope will help to detect and correct deviations between the driver's intended course and the actual course of the vehicle. Such deviations will be determined through the use of steering column sensors to measure intended course, and yaw rate sensors that measure the car's actual course by detecting the degree of vehicle spin or rotation. The latest models of Cadillacs, Corvettes, Mercedes, BMWs, Volkswagens will be using rate sensors in vehicle dynamic control systems that will improve car safety. ${ }^{2}$

\subsection{Navigation and GPS Augmentation}

MEMS gyroscopes and accelerometers can potentially be used to form an inertial navigation unit to augment GPS.

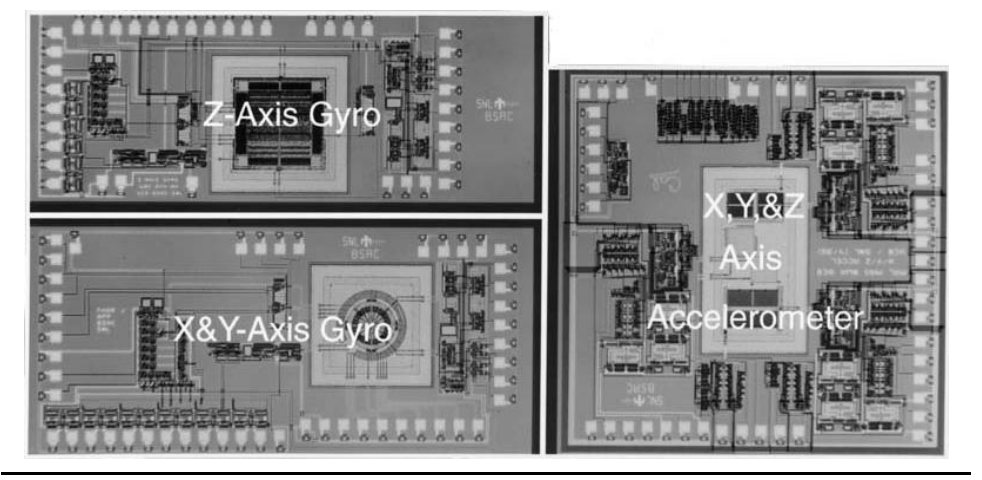

Figure 3. A six-degree-of-freedom, fingernail-sized IMU with integrated signal-processing and control circuitry, designed at the University of California, Berkeley, and fabricated by Sandia National Laboratories (Courtesy of BSAC).

Shown in Fig. 3 is a prototype of a six-degree-of-freedom inertial measurement unit (IMU) on-a-chip with integrated signal-processing and control circuitry, which is designed at the University of California, Berkeley, and fabricated by Sandia National Laboratories. This unit combines a dual-axis rate gyroscope, a z-axis gyroscope, and a three-axis accelerometer chip to measure angular rate and acceleration without the need to align individual sensors.

MEMS gyroscopes, however, are currently immature and require several orders of magnitude improvement in stability. To be useful in GPS augmentation, the gyro drift rate should be low enough to make the device useful for operations of at least 2-4 hours between GPS position updates. This is currently a very big challenge for micromachined gyroscopes.

\subsection{Consumer Electronics}

There is a wide range of applications for small and inexpensive angular rate sensors in consumer electronics. Applications that are already entering the market are gyro-based anti-jitter system for comcorders, 3D computer air mouse, wireless $3 \mathrm{D}$ pointing devices for interactive presentations. Low-cost gyroscopes are also entering toy and game industry markets; currently advertised are gyro-enhanced wireless remote-control cars, motion feedback systems for video games and virtual reality. Presently, micro-machined gyroscopes are able to meet cost and performance requirements for most of consumer electronics applications.

\subsection{Robotics and Intelligent Systems}

There is also a large potential for using low-cost gyroscopes for robotic systems, from industrial cranes to intelligent mobile robots and arm-manipulators. In case of mobile robots, MEMS gyroscopes and accelerometers, in conjunction with a compass and inclinometers, can provide reliable position and orientation estimation in robot navigation tasks. As described earlier, MEMS Inertial Measurement Units can be an essential tool for position measurements in robot navigation where GPS is not available or obscured (e.g., indoor applications, other planets, or near the poles).

Other potential applications of low-cost gyroscopes are location units for handling inventory in large warehouses and industrial plants and personal navigation systems for electronic map following.

Micromachined gyroscopes can also be successfully used for stabilization of satellite antenna systems of shipboards, aircrafts and land vehicles. Cost-effective miniature gyroscopes can be incorporated into the newest generation of high-reliability, solid-state avionics and flight information systems. 


\section{THE CORIOLIS EFFECT AND \\ PRINCIPLE OF OPERATION}

Most micromachined gyroscopes are utilizing the concept of using a vibrating element to measure rotational velocity based on the Coriolis principle.

In most designs, the micromachined vibrating angular rate sensor consists of a mass suspended on elastic supporting flexures anchored to the substrate. The devices are typically fabricated using lithography, deposition, and sequential chemical etching of polysilicon film and release film, as well as shaping devices from a single silicon wafer or monocrystalline piezoelectric quartz.

In the basis of operation, the proof-mass, which constitute the active portion of the sensor, is driven by an oscillator circuit at a precise amplitude and high frequency, Fig. 4. When subjected to a rotation, the proof-mass will be subjected to the Coriolis force:

$$
F=2 m \Omega \times V_{c}
$$

where $m$ - mass, $V_{c}$ - instantaneous radial velocity of the center of mass, $\Omega$ - input rate.

To describe dynamics of the gyroscope, it is convenient to introduce two coordinate systems: $\{\xi, \eta, \zeta\}-$ inertial, fixed in an absolute space, and $\{x, y, z\}$ - non-inertial, rigidly attached to the platform. Angular velocity $\vec{\Omega}=$ $\left(\Omega_{x}, \Omega_{y}, \Omega_{z}\right)$ of the platform is associated with angular velocity of the object to which the gyroscope is attached, Fig. 4(a).

The behavior of the gyroscope is naturally described with respect to the non-inertial coordinate frame $\{x, y, z\}$. When equations of motion are written with respect to this frame, the "fictitious" or "inertial forces" has to be treated as actual physical forces. The second Newton Law then has the form $m \vec{a}_{x y z}=\vec{F}_{e x t}-m \vec{a}_{e}-m \vec{a}_{c}$. Here, $\vec{a}_{x y z}$ is a linear acceleration of the gyroscope with respect to the coordinate frame $\{x, y, z\}, \vec{F}_{e x t}$ is a sum of all external forces acting on the proof mass (including elastic restoring forces, damping, etc.), $\vec{a}_{e}$ is centrifugal acceleration which is a function of $\Omega^{2}$ and $\dot{\vec{\Omega}}$, and $\vec{a}_{c}$ is the Coriolis acceleration.

In traditional applications of gyroscopes the rotation rate is small relative to the natural frequency of the system and is also constant over a relatively long time interval. Also, linear acceleration terms can be typically cancelled out as an offset from the output response. In addition, if we are interested in measuring the rotation rate about only the z-axis (Fig. 4), then it is possible to make the stiffness in the z-direction much larger than the stiffness in the other two orthogonal directions through special fabrication techniques and careful design. If all these requirements are satisfied, all inertial forces, except the Coriolis force, can be ignored (or compensated) and the governing equations in Cartesian coordinates $\{x, y, z\}$ are given by

$$
\begin{aligned}
& \ddot{x}+\omega_{n}^{2} x-2 \Omega \dot{y}=0 \\
& \ddot{y}+\omega_{n}^{2} y+2 \Omega \dot{x}=0
\end{aligned}
$$

The essential feature of these equations is the presence of the Coriolis acceleration terms $-2 \Omega \dot{y}$ and $2 \Omega \dot{x}$. These two terms will appear only if the equations of motion are written in a non-inertial coordinate frame. It is the Coriolis acceleration that causes a transfer of energy between two of the gyroscope modes of operation.

The resultant Coriolis force is perpendicular to both the input rate and the instantaneous radial velocity in the drive direction. This produces a motion of the proof-mass in direction perpendicular to its initial oscillation.

The pickup combs (or electrodes), being the sensing portion of the micromachined gyroscope, respond to the oscillation of the proof-mass by producing an electrical signal. After amplification, this signal is demodulated into a DC signal proportional to the sensor's rotation.

To measure rotation rate, the proof-mass is driven to a fixed amplitude along the $\mathrm{x}$-axis by applying an electrostatic drive force to the proof-mass along the x-axis. In the absence of rotation there will be no motion of the proof-mass along the y-axis, Fig. 4(a). Under rotation, however, the Coriolis acceleration will cause energy to be transferred from the $\mathrm{x}$-axis (primary mode) to the $\mathrm{y}$-axis (secondary mode) building up a vibration amplitude along the $\mathrm{y}$-axis. 


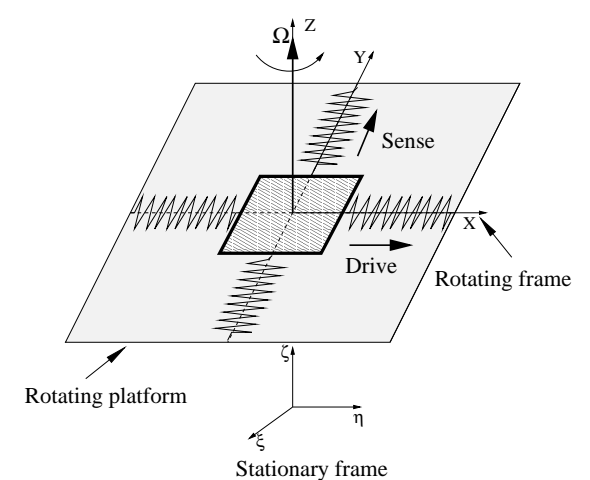

(a)

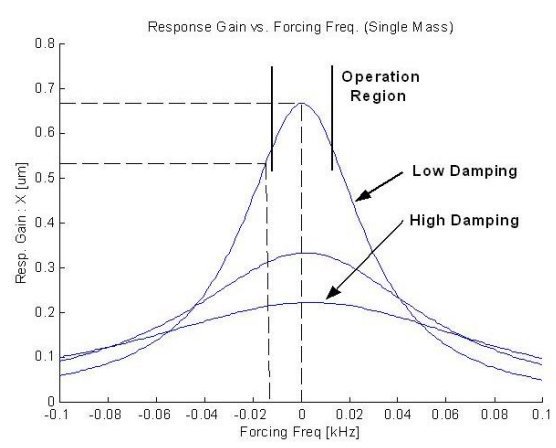

(b)

Figure 4. (a) Mass-spring model of the vibratory micromachined gyroscope; (b) The response of the vibratory gyroscope to the Coriolis force.

The ratio of the amplitude in the secondary mode of vibration to the amplitude of the primary mode of vibration can be shown to be proportional to the rotation rate and is given by

$$
\frac{y}{x}=2 Q \frac{\Omega}{\omega_{n}}
$$

Notice, that the gyroscope response is proportional to quality factor $Q$ of the device. If the quality factor is not changing, the rotation rate can be determined by simply measuring the amplitude of the secondary mode, Fig. 4(b). Rotation rate can be measured by operating the gyroscope in either the open-loop or the closed-loop (force-torebalance) modes. In the closed loop mode, the control system operates exactly like the amplitude control in the drive direction. Only in the case of the closed-loop control, the secondary mode amplitude is continuously driven to zero rather than to a fixed value.

\section{RESEARCH AND DEVELOPMENT EFFORTS}

World-wide, many academic universities, research centers, and industrial laboratories make efforts to develop high quality cost-effective inertial sensors which could replace their existing bulky and very expensive counterparts. To achieve this goal, micromachined silicon gyroscopes have been the subject of extensive research and development over the past few years (a comprehensive review was recently published $\mathrm{in}^{3}$ ).

A number of vibratory gyroscopes have been demonstrated, including tuning forks, ${ }^{4}$ vibrating beams, ${ }^{5}$ and vibrating shells. ${ }^{6}$ Achieving high-grade performance levels has proven to be a tough challenge for micromachined gyroscopes, and new technologies and approaches are being developed.

\subsection{Microfabrication Technologies}

Quartz based technologies were the first technologies employed in the fabrication of cost effective gyroscopes. In the late 1980's, after successful demonstration of batch-fabricated silicon accelerometers, some efforts were initiated to replace quartz with silicon in micromachined vibratory gyroscopes.

In general, silicon micromachining processes for fabrication of vibratory gyroscopes fall into one of four categories: 1) polysilicon surface micromachining; 2) silicon bulk micromachining and wafer bonding; 3) combined bulk and surface micromachining or so-called mixed processes; 4) Quartz-based processes.

\subsection{Surface Micromachined Gyroscopes}

Silicon surface micromachining is the most advanced technology for manufacturing sensors on-a-chip. This is mainly due to its compatibility with a well established conventional IC technology. In recent years there a number of surface micromachining fabrication processes have been developed which, in addition to shrinking the sensor size by several orders of magnitude in comparison to conventional sensors, allow to add integrated electronics to the same silicon chip, creating an entire system on a chip. ${ }^{7}$ Below we review some of the results in gyroscope development using surface micromachining technologies. 


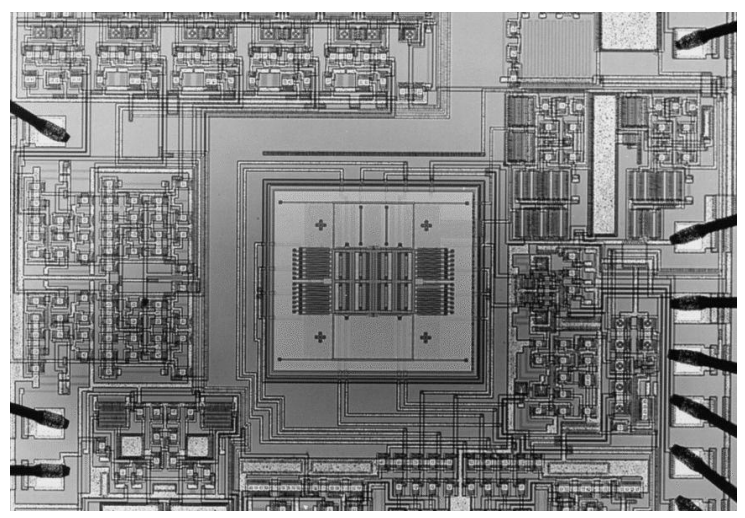

Figure 5. Berkeley's Z-axis vibratory rate gyroscope was integrated with a transresistance amplifier on a single die using the Analog Devices BiMEMS process ${ }^{8}$

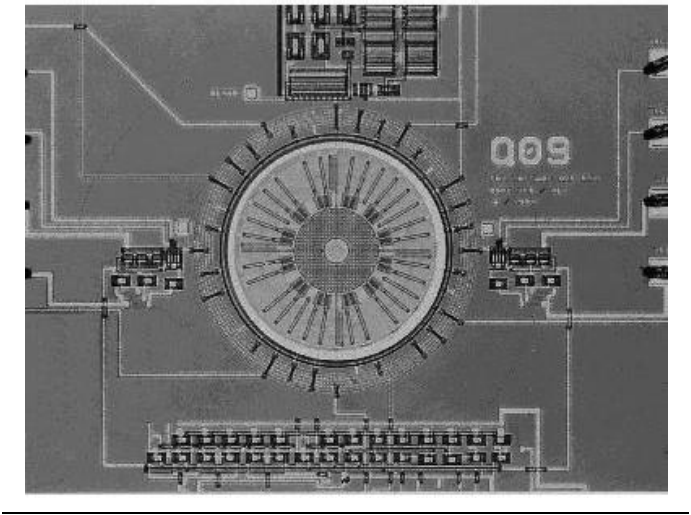

Figure 6. Die shot of Berkeley's Dual-axis rate gyroscope, integrated with sense and drive electronics using Analog Devices, Inc., surface-micromachining process.$^{9}$

Single- and dual- axis polysilicon surface micromachined gyroscopes have been realized by researchers at Berkeley, ${ }^{8}$ ${ }^{9}$ and Samsung. ${ }^{10-12}$ Berkeley's Z-axis vibratory rate gyroscope was integrated with a trans-resistance amplifier on a single die using the Analog Devices BiMEMS process, Fig 5 . This device demonstrated a resolution of $1^{0} / s / \sqrt{H z}$ with performance projected to improve to $0.1^{0} / s / \sqrt{H z}$.

Samsung has also reported a very similar surface-micromachined z-axis device, that demonstrated a resolution of $0.1^{0} / s$ at $2 \mathrm{~Hz}$, an operating bandwidth of $100 \mathrm{~Hz}$, and a linearity of $1 \%$ full scale in a range of $90^{\circ} / \mathrm{s}$.

Berkeley has reported an interesting surface micromachined dual-axis gyroscope which is based on rotational resonance of a $2-\mu m$-thick polysilicon rotor $\operatorname{disk}^{9}$ (Fig. 6). This device, integrated with electronics, yielded a random walk as low as $10^{\circ} / \sqrt{\mathrm{Hz}}$ with cross-axis sensitivity ranging $3-16 \%$.

Murata has demonstrated a surface-micromachined polysilicon gyroscope that is sensitive to lateral (x-or y-axis) angular rate. ${ }^{13}$ This device showed an open-loop noise-equivalent rate of $2^{0} / s / \sqrt{H z}$. Later in 1997, Samsung reported a similar device. This device showed an improved open-loop noise-equivalent rate of $0.1 / s / \sqrt{H z}$ with a good linearity up to $100^{\circ} / \mathrm{s}$.

Researchers at General Motors and the University of Michigan have developed a vibrating ring gyroscope ${ }^{6}$ using the technology of electroforming nickel into a thick polymide (or photoresist) mold on a silicon substrate in a post circuit process. The gyroscope demonstrated a resolution $0.5^{0} / \mathrm{s}$ in a $25 \mathrm{~Hz}$ bandwidth.

Recently, a group at HSG-IMTT, Germany, have demonstrated and reported a surface-micromachined precision $\mathrm{x}$-axis vibratory gyroscope (MARS-RR) with standard Bosch foundry process featuring a $10 \mu m$ thick structural polysilicon layer in addition to the buried polysilicon layer, which defines the sense electrodes. The reported performance of this device is quite impressive: a random angle walk of $0.27^{0} / \sqrt{h}$, a bias stability of $65^{0} / h$, and a scale-factor nonlinearity of $<0.2 \% .^{14}$ 


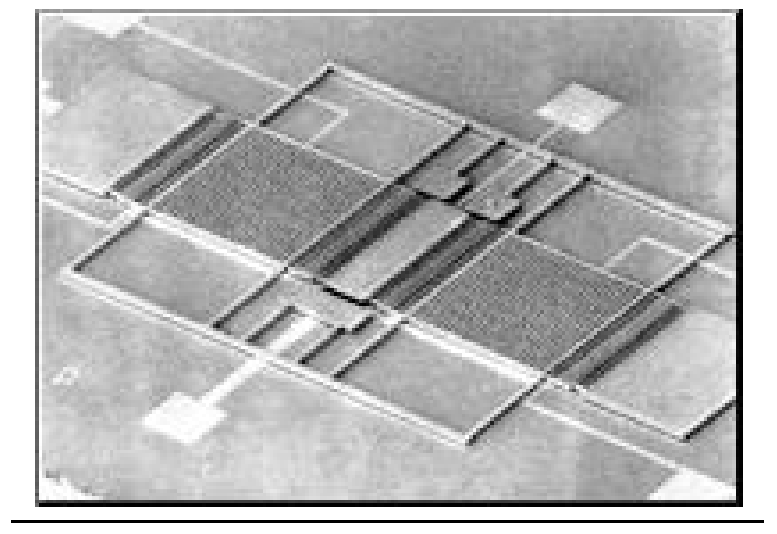

Figure 7. Draper's single-crystal silicon-on-glass tuning-fork gyroscope ${ }^{4}$

\subsection{Bulk-Micromachined Gyroscopes}

The Charles Stark Draper Laboratory demonstrated one of the first batch-fabricated silicon micromachined rate gyroscopes in 1991. This bulk silicon device was a double gimbal vibratory gyroscope supported by torsional flexures, with the vibrating mechanical element made from $\mathrm{p}++$ silicon (100). A rotation rate resolution of $4^{0} / s$ in a $1 \mathrm{~Hz}$ bandwidth was realized using this structure. Later in 1993, Draper reported an improved $1 \mathrm{~mm}^{2}$ silicon-on-glass tuning fork gyroscope, Fig. 7, fabricated through the dissolved wafer process. ${ }^{4}$ The noise equivalent rate observed by this structure was $470^{\circ} / h$ in a $60 \mathrm{~Hz}$ bandwidth, equivalent to $0.02^{\circ} / \mathrm{s}$ in a $1 \mathrm{~Hz}$ bandwidth or angle random walk of $0.72^{0} / \sqrt{h}$. With a thermal control integrated on-a-chip this device is projected to improve performance to $10^{0} / h$.

The JPL, in collaboration with the University of California, Los Angeles, has demonstrated a bulk micromachined, precision silicon MEMS vibratory gyroscope for space applications. ${ }^{15}$ A bias stability of $70^{\circ} / h$ and an angle random walk of $6.3^{\circ} / \sqrt{h}$ were achieved in this device. It should be noticed, however, that this device is non-monolithic and requires an after-fabrication assembling.

Researchers at the University of Neuchatel, Switzerland, have demonstrated a tuning-fork design. ${ }^{16}$ This gyroscope was fabricated through silicon bulk micromachining and was wafer-level vacuum packaged by anodic bonding of the silicon wafer to encapsulate glass wafers. This device showed a sensitivity of $4 \mathrm{nV} / \% / \mathrm{s}$ with excellent linearity up to $750^{\circ} / \mathrm{s}$.

British Aerospace Systems and Equipment, in collaboration with Sumitomo Precision Products, has developed a micromachined single-crystal silicon ring gyroscope with a reported root-mean-square noise floor of $0.15^{0} / s$ in a 30 $\mathrm{Hz}$ bandwidth and an in-run drift of approximately $0.05 / \mathrm{s} .{ }^{17}$ This device was fabricated through deep dry etching of a 100- $\mu m$-thick silicon wafer, which was then anodically bonded to a glass support wafer.

\subsection{Gyroscopes Fabricated Using Mixed Processes}

Bosch proposed a gyroscope fabricated through a combination of bulk- and surface- micromachining processes. ${ }^{18}$ This silicon yaw rate sensor achieves vibration amplitudes as large as $50 \mu m$ using a permanent magnet mounted inside a metal package. Operating at atmospheric pressure, the device has shown a resolution of $0.3^{0} / s$ in $100 \mathrm{~Hz}$ bandwidth.

Daimler Benz has demonstrated a tuning-fork angular rate sensor for automotive applications that piezoresistively measures the rotation-induced shear stress in the stem of the tuning-fork device. ${ }^{19}$ This device was fabricated through a combination of bulk micromachining and bonding of SOI wafers.

Currently, UC-Berkeley, MIT, and Honeywell Technology Center are developing a highly sensitive, monolithic silicon gyroscope using a high aspect-ratio single-crystal silicon integrated MEMS technology.

\subsection{Quartz Technology}

Piezoelectric vibratory gyroscopes were demonstrated in the early 1980's. Examples of these devices are fussed quartz HRG by Delco, quartz tuning forks like the Quartz Rate Sensor by Systran Donner, and a piezoelectric vibrating disc gyro. Although quartz vibratory gyroscopes can yield very high quality factors at atmospheric pressure with improved level of performance, their batch processing is not compatible with IC fabrication technology. 


\section{CHALLENGES}

\subsection{Zero Rate Output}

An important performance parameter for a vibratory gyroscope is its zero rate output (or zero bias). Geometrical imperfections in the vibrating mechanical structure and/or the sense and drive electrodes as well as electrical coupling between these electrodes can cause an output signal in the absence of rotation. For instance, in tuning-fork designs, if due to fabrication flaws the tines' centers of mass are not precisely aligned in the plane of vibration, their inertial forces produce a vibrating torque about the stem just as the Coriolis torque does. This error, often called the quadrature error, can be distinguished from the rate signal simply because it is in phase quadrature with the Coriolis-induced signal. If too large, it may cause errors in sensing the rotation rate. In the case of shell gyroscope designs, asymmetric damping of the structure, which manifests itself as different sense and drive mode quality factors, can also cause a finite output when there is no rotation. By electrically and mechanically decoupling the sense and drive modes, and by minimizing the fabrication process errors, zero rate output (ZRO) can be significantly reduced. A large quality factor and lower natural frequency can further reduce the ZRO, and its drift simply due to the fact that these two factors increase the open-loop sensitivity of the sensor. Therefore, high-quality materials with low internal damping - such as silicon, germenium, or quartz - will improve the accuracy of the sensor. Any remaining zero bias error should then be further reduced electronically.

Fortunately, the mechanically coupled acceleration is $90^{\circ}$ phase displaced with respect to the velocity induced Coriolis acceleration and can be a synchronous demodulator to the extent of its phase accuracy. However, this rejection is limited. So, the quality of the mechanical design is measured by the size of the interfering $90^{\circ}$, or "quadrature" signal it produces from manufacturing tolerances. High aspect ratio flexures can be very valuable in improving those tolerances. Also, control of the flexure sidewall angels was recognized as critically important even in the bulk etched devices with very large aspect ratios.

\subsection{Matching Drive and Sense Frequencies}

If the sense and drive resonant modes of a tuning fork have equal frequencies, the output signal will be amplified by the quality factor $\mathrm{Q}$ of the sense mode, resulting in much higher sensitivity. However, this involves extreme control of device dimensions and may lead to temperature drift problems if these natural frequencies do not track it with temperature. Because of these difficulties, most tuning-fork designs are not based on matched vibration mode frequencies. Another solution which would allow to decrease sensitivity of the system to parameters variation would be to increase the number of DOF. This would expand the design space and allow to find a set of system parameters which would widen the bandwidth of the system.

\subsection{Noise and Minimal Measured Signal}

The miniaturization is an important advantage of MEMS, which allows, for example, significantly reduce device's power consumption (e.g., allows to operate from a small battery and be portable). However, any significant size reduction of gyroscopes is accompanied by a loss of their performance. The performance of MEMS vibratory gyro is defined eventually by minimum detectable angular rate. Therefore, this problem should be solved by optimal designing: a selection of low noise elements in signal transforming chain, a providing favorable conditions of their maximal robustness to external noise, an increasing the output signal value at any given input angular rate. This will be possible by providing effective use of favorable electrical and mechanical properties of silicon as design material, as well as providing near resonant oscillations of proof mass at high values of Q-factor of exciting and sensing oscillation contours. The microstructure also can be made as thick and narrow as possible, justifying the need for high aspect ratio microstructures.

\section{DESIGN SOLUTIONS}

\subsection{Rate Gyro with Structural Disturbance Rejection}

An interesting solution to compensate for quadrature errors was proposed by Analog Devices Inc. ${ }^{20}$ In their design, the gyro has a suspended body with an inner frame and an outer frame surrounding inner frame Fig. 8(a). Frames in this design are coplanar and are suspended over and parallel to an underlying substrate. Outer frame is suspended with flexures that extend along axes parallel to a dither axis and are anchored to substrate. This arrangement of flexures allows outer frame to move along a Coriolis axis, but substantially prevents outer frame from moving along dither axis. 


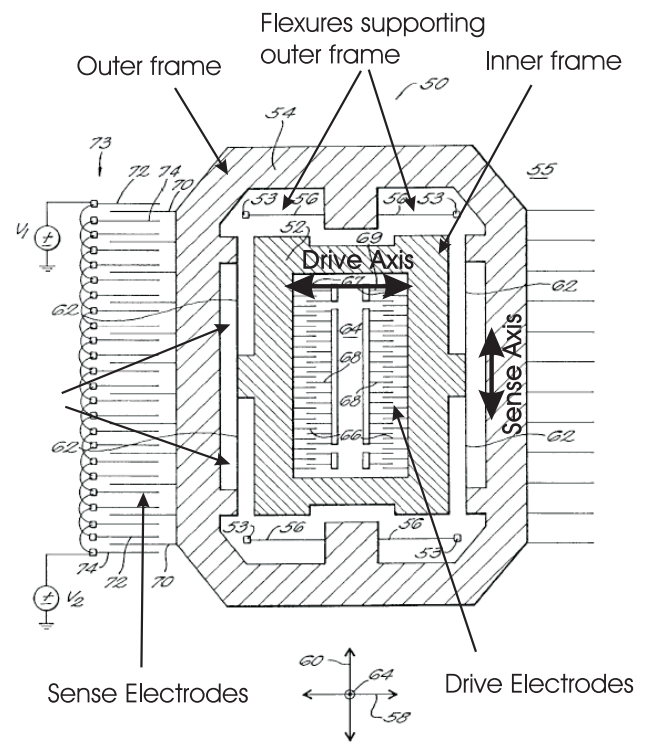

(a)

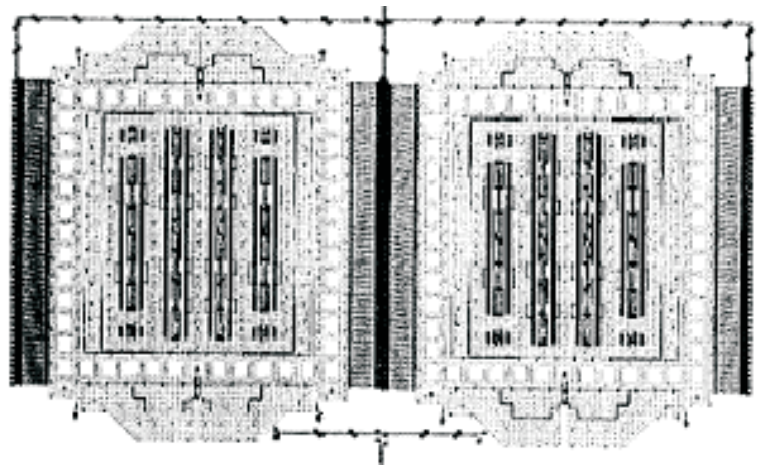

(b)

Figure 8. A conceptual design of a gyroscope proposed by Analog Devices Inc. ${ }^{20}$

Inner frame is coupled to and suspended from outer frame with flexures that extend along axes parallel to Coriolis axis. The orientation of flexures allows inner frame to move along dither axis relative to outer frame, substantially inhibits relative motion of the frames along Coriolis axis, but allows inner frame and outer frame to move together along Coriolis axis. Accordingly, for both inner frame and the outer frame, control over allowable and inhibited directions of movement is achieved by orienting the axes of the flexures along the inhibited axis. ${ }^{20}$

The sensor Fig. 8(b) has been fabricated using 2 microns surface micromachining technology. ${ }^{21}$ The device has nearly 5000 fingers, has a $\mathrm{Q}$ of 20 to $10 \mathrm{kHz}$, and vibrates with an amplitude of 9 microns. According to, ${ }^{21}$ the sensor has an impressive, less than $1 \mathrm{ppm}$, quadrature errors. No results on sensitivity and performance of the device have been reported yet.

\subsection{Rate Gyro with Dynamic Disturbance Rejection}

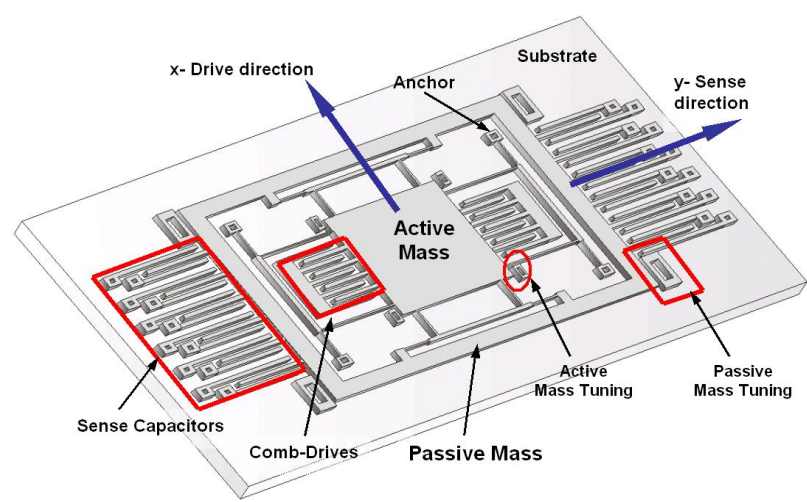

(a)

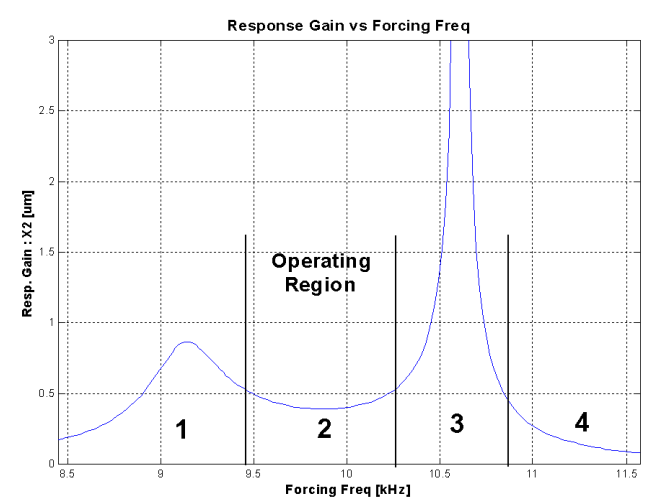

(b)

Figure 9. (a) Illustration of dual-mass z-axis gyroscope with extended bandwidth; (b) Response of the dual-mass gyroscope in the flat operation region is insensitive to resonant frequency fluctuations and has over 15 times wider bandwidth than existing gyroscopes.

All existing micromachined rate gyroscopes operate on the vibratory principle of a single proof mass suspended by flexures anchored to the substrate. To achieve the maximum possible gain, the conventional gyroscopes are designed 


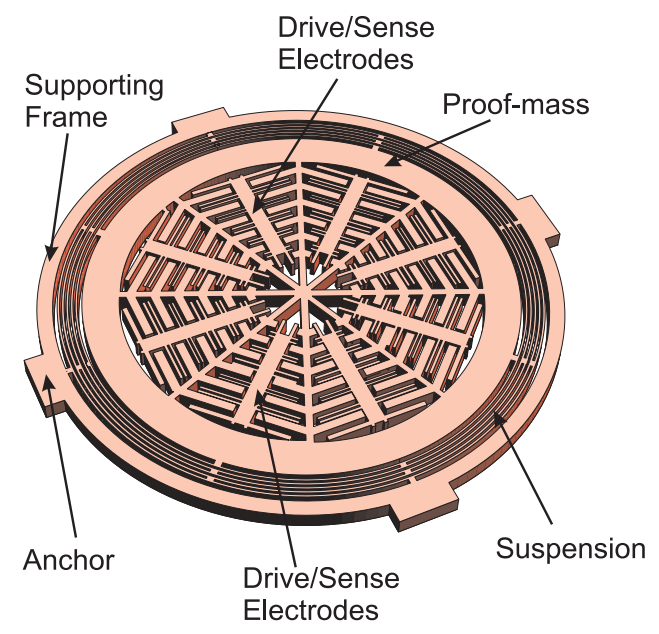

(a)

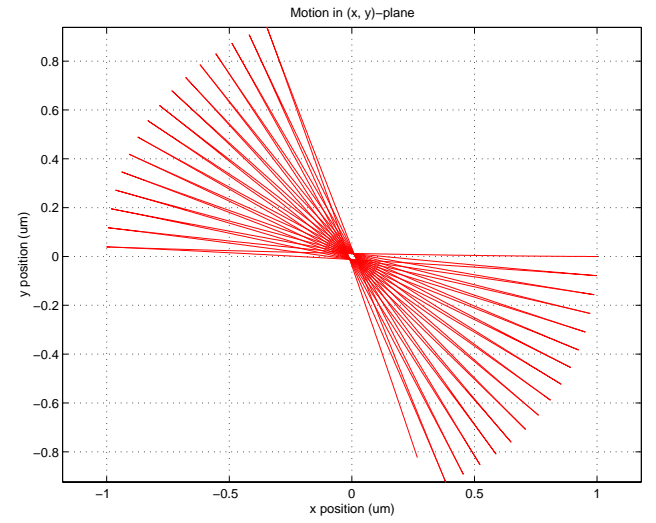

(b)

Figure 10. The Coriolis acceleration causes the precession of the line of oscillation. The oscillating proof mass is intending to keep the line of oscillation constant in the absolute space; this effect is equivalent to the transfer of energy between two of the gyroscope modes of operation in a non-inertial coordinate frame. With the help of adaptive control electronics the gyroscope will keep the energy level constant at all time. The asotropy of the devices and appropriate control architecture will allow to the vibration pattern to precess.

to operate at the peak of the response curve. This is typically achieved by matching drive and sense resonant frequencies. However, the system is very sensitive to variations in system parameters causing a shift in the resonant frequency. For example, a $1 \%$ fluctuation in frequency matching between drive and sense modes will produce an error of $20 \%$ in the output signal gain. ${ }^{22}$ Under high quality factor conditions the gain is high, however the bandwidth is extremely narrow. In addition, the gain is affected significantly by fluctuations in damping conditions, Fig. 4(b).

Fabrication imperfections are inevitable, and affect material properties and geometry of MEMS structures. For surface micromachining, the thickness of the suspension elements is determined by deposition process, and the width is affected by etching process. Variations in elastic modulus, beam thickness or residual stresses have drastic effects on dynamic response of gyroscopes. Generally, very sophisticated control electronics is used to provide operation in the region of the resonance peak. ${ }^{23}$ Furthermore, during the operation time of these devices, fluctuations in the ambient temperature alter the gyroscope geometry together with structural properties, and pressure fluctuations affect the damping conditions; resulting in significant errors.

To eliminate the limitations of the existing micromachined gyroscopes, a design approach that does not require the system to operate in resonance can be effectively used.$^{24}$ The proposed architecture suggests the use of two independently vibrating proof masses in the dynamical system (Fig. 9(a)) instead of one, as this is typically done in the conventional devices. The first mass is forced to oscillate in the drive direction, and this forced oscillation is amplified by the second mass. The response of the second mass in the orthogonal sense direction is monitored.

By utilizing dynamical amplification design idea, the necessity of operation in the resonance mode is eliminated, and over 15 times increase in the bandwidth of the system is achieved. ${ }^{25,26}$ The proposed device is demonstrated to have improved robustness against expected fabrication and packaging fluctuations, especially against damping variations due to ambient pressure, compared to the conventional micromachined gyroscopes. Sensitivity analysis revealed that, for the same thermal loading, the device produces $87 \%$ less error than conventional gyroscopes. Moreover, the proposed design was shown to be approximately 12 times less sensitive to residual stresses, and 20 times less sensitive to fabrication variations than conventional gyroscopes. Consequently, with the presented design approach, tight fabrication tolerances and packaging requirements can be relaxed resulting in a lower production cost of MEMS gyroscopes.

\subsection{MEMS Angular Gyroscope}

The vibratory gyroscopes can potentially operate in two different modes: the angle and the angular rate modes. ${ }^{23}$ All existing micromachined gyroscopes, however, can only operate in the angular rate measuring mode. Recently, 
there was proposed a design concept for MEMS gyroscopes that will allow to measure the angle of rotation directly. ${ }^{27}$ The proposed device utilizes a vibrational "lumped mass-spring system" with electrostatic combs used for drive and sense Fig. 10(a). The use of sense combs interwoven between the comb fingers of the proof mass increases the sense capacitance, and therefore the sense capabilities, of the device. Upon reaching the desired amplitude, the drive force is removed and the energy of the system is maintained using a specially designed control architecture. The rotation induced Coriolis force causes the line of oscillation to precess and the precession angle is proportional to the angular displacement of the device.

When the isotropic oscillator is allowed to freely oscillate, the precession of the straight line of oscillation provides a measure of the angle of rotation (Fig. 10). The angle of the precessing pattern can be instantly defined from the measurements of displacement and velocity vectors ${ }^{23}$ :

$$
\tan \phi=\frac{2\left(\omega_{n}^{2} x y+\dot{x} \dot{y}\right)}{\omega_{n}^{2}\left(x^{2}-y^{2}\right)+\left(\dot{x}^{2}-\dot{y}^{2}\right)}
$$

Here, $\phi=\int \Omega \cdot d t$ is the angle of rotation, $\omega_{n}$ is the fundamental frequency of oscillation, $x$ and $y$ are the linear displacements of the proof-mass with respect to the reference coordinate system $\{X, Y, Z\}$.

It has been demonstrated that the device has necessary isotropy required for the operation of the device. ${ }^{28,29}$ In contrast to classical approach, thermal analysis of the angular gyroscope confirms that the sensor is more robust and significantly more resilient to temperature variations and induced residual stress.

\section{SUMMARY}

Until recently, there was no lightweight, inexpensive, reliable inertial sensor that could be used to directly measure rotational velocity. Traditional spinning wheel gyroscopes were heavy, consumed a lot of power, and, because they had moving mechanical parts, wore out after just a few thousand hours of operation.

A demand for silicon based inertial sensors raised, which promises a new wide market for many areas. In order to introduce the inertial technology into the market, it is necessary to meet a number of principal requirements: significant cost reduction of fabrication, testing and packaging; decreasing of power consumption by miniaturization; enhancing of performance and reliability by careful design; and on-chip integration of device with electronics for multiplicity and ultimate cost reduction. In spite of all obstacles discussed above, however, a great efforts of developing inertial sensors will enable to commercialize these devices in a year or two years.

\section{ACKNOWLEDGEMENTS}

The author would like to thank Roger T. Howe and Roberto Horowitz, both from the University of California at Berkeley, as well as Cenk Acar and Chris Painter, from the University of California at Irvine, for their contributions.

\section{REFERENCES}

1. K. Gabriel, "Microelectromechanical Systems Program: Summary of Research Activities", Advanced Research Projects Agency, 1994.

2. A. M. M. et al., "A miniature yaw rate sensor for intelligent chassis control," Proc ITSC'97, IEEE Conf on Intelligent Transportation Systems, 1997. 912 Nov. Boston, MA, Paper No. 0002.

3. Navid Yazdi, Farrokh Ayazi, and K. Najafi, "Micromachined inertial sensors," Proceedings of the IEEE 86, pp. 1641-1659, Aug. 1998.

4. J. Bernstein, S. Cho, A.T. King, A. Kourepenis, P. Maciel, and M. Weinberg, "A micromachined comb-drive tuning fork rate gyroscope," IEEE Micro Electro Mechanical Systems Workshop (MEMS'93), pp. 143-148, Feb. 1993. Fort Lauderdale, Florida.

5. K. Maenaka and T. Shiozawa, "A study of silicon angular rate sensors using anisotropic etching technology," Sensors and Actuators A 43, pp. 72-77, 1994.

6. M. Putty and K. Najafi, "A micromachined vibrating ring gyroscope," IEEE Solid State Sensors and Actuators Workshop, pp. 213-220, June 1994. Hilton Head Island, SC.

7. J. H. Smith, J.J. Sniegowski, P.J. McWhorter, and A. R. Jr., "Intelligent microsystems: Strategy for the future," Semiconductor Int. 21, pp. 93-98, 1998. 
8. W. A. Clark, R. T. Howe and R. Horowitz, "Surface micromachined z-axis vibratory rate gyroscope," IEEE Solid State Sensors and Actuators Workshop, pp. 283-287, June 1996. Hilton Head Island, SC.

9. T. Juneau and A. Pisano, "Micromachined dual input axis angular rate sensor," IEEE Solid State Sensors and Actuators Workshop, pp. 299-302, June 1996. Hilton Head Island, SC.

10. Y. Oh, B. Lee, S. Baek, H. Kim, J. Kim, S. Kang, and C. Song, "A surface-micromachined tunable vibratory gyroscope," IEEE Micro Electro Mechanical Systems Workshop (MEMS'97), pp. 272-277, 1997. Japan.

11. K.Y. Park, C.W. Lee, Y.S. Oh, and Y. H. Cho, "Laterally oscillated and force-balanced micro vibratory rate gyroscope supported by fish hook shape springs," IEEE Micro Electro Mechanical Systems Workshop (MEMS'97) , pp. 494-499, 1997. Japan.

12. S. An, Y.S. Oh, B.L.Lee, K.Y.Park, S.J.Kang, S.O.Choi, Y. I. Go, and C. Song, "Dual-axis microgyroscope with closed-loop detection," IEEE Micro Electro Mechanical Systems Workshop (MEMS'98), pp. 328-333, 1998. Heidelberg, Germany.

13. K. Tanaka, Y. Machida, M. Sugimoto, K. Moriya, T. Hasegawa, K. Atsuchi, and K. Ohwada, "A micromachined vibrating gyroscope," Sensors and Actuators A 50, pp. 111-115, 1995.

14. H. J. Merz, "A new silicon rate gyroscope," IEEE Micro-Electromechanical Systems Workshop (MEMS'98), 1998. Heidlberg, Germany.

15. T.K. Tang, R.C. Gutierrez, J.Z. Wilcox, C. Stell, V. Vorperian, R. Calvet, W.J. Li, I. Charkaborty, R. Bartman, and W. Kaiser, "Silicon bulk micromachined vibratory gyroscope," Tech. Dig. Solid-State Sensor and Actuator Workshop , pp. 288-293, 1996. Hilton Head Island, SC, June.

16. F. Paoletti, M.A. Gretillat, and N. F. de Rooij, "A silicon micromachined vibrating gyroscope with piezoresistive detection and electromagnetic excitation," IEEE Micro Electro Mechanical Systems Workshop (MEMS'96), pp. 162-167, 1996. San Diego, CA.

17. I. Hopkin, "Performance and design of a silicon micromachined gyro," Proc. Symp. Gyro Technology, pp. 1.01.10, 1997. Stuttgard, Germany.

18. M. Lutz, W. Golderer, J. Gerstenmeier, J. Marek, B. Maihofer, S. Mahler, H. Munzel, and U. Bischof, "A precision yaw rate sensor in silicon micromachining," Tech. Dig. Solid-State Sensor and Actuator Workshop (Transducers'97) , pp. 847-850, 1997. Chicago, IL, June.

19. R. Voss, K. Bauer, W. Ficker, T. Gleissner, W. Kupke, M. Rose, S. Sassen, J. Schalk, H. Seidel, and E. stenzel, "Silicon angular rate sensor for automotive applications with piezoelectric drive and piezoelectric read-out," Tech. Dig. Solid-State Sensor and Actuator Workshop (Transducers'97), pp. 879-882, 1997. Chicago, IL, June.

20. J. A. Geen, "Micromachined Gyros", Patent Number US-6,122,961, 2000.

21. J. A. Geen, "A Path to Low Cost Gyroscopy," IEEE Solid State Sensors and Actuators Workshop , pp. 51-54, June 1998. Hilton Head Island, SC.

22. E. Netzer and I. Porat, "Novel vibratory device for angular rate measurement," Journal of Dynamic Systems, Measurement and Control, Dec. 1995.

23. A. Shkel, R. Horowitz, A. Seshia, and R. T. Howe, "Dynamics and control of micromachined gyroscopes," The American Control Conference, June 1999. San Diego, CA. (Invited Paper).

24. C. Acar and A. M. Shkel, "Wide Bandwidth Micromachined Gyroscope to Measure Rotation, Patent pending, UCI Office of Technology Alliances, Case No:2001-140-1, 2000.

25. C. Acar and A. M. Shkel, "A design approach for robustness improvement of rate gyroscopes," The Fourth International Conference on Modeling and Simulation of Microsystems (MSM 2001), 2001. Hilton Head Island, SC.

26. C. Acar and A. M. Shkel, "Micro-gyroscopes with Dynamic Disturbance Rejection," 2001 SPIE Annual International Symposium on Smart Structures and Materials, pp. 4334-11, 2001. Newport Beach, CA.

27. A. Shkel and R. T. Howe, "Surface micromachined rate integrating gyroscopes," Patent Pending Application, Dec. 1998. UC-Berkeley Office of Technology and Licensing.

28. C. Painter and A. M. Shkel, "Study of a Novel Isotropic Suspension Design for an Angular Gyroscope," The Fourth International Conference on Modeling and Simulation of Microsystems (MSM 2001), 2001. Hilton Head Island, SC.

29. C. Painter and A. M. Shkel, "Structural and Thermal Analysis of a MEMS Angular Gyroscope," 2001 SPIE Annual International Symposium on Smart Structures and Materials , pp. 4334-09, 2001. Newport Beach, CA. 